\title{
The new modified ABCD method for gastric neoplasm screening
}

\author{
Chan Hyuk Park · Eun Hye Kim • Da Hyun Jung • \\ Hyunsoo Chung $\cdot$ Jun Chul Park $\cdot$ Sung Kwan Shin • \\ Sang Kil Lee $\cdot$ Yong Chan Lee
}

Received: 9 December 2014 / Accepted: 25 January 2015/Published online: 8 February 2015

(C) The International Gastric Cancer Association and The Japanese Gastric Cancer Association 2015

\begin{abstract}
Background The ABCD screening method was developed for risk stratification of gastric cancer. It is unclear whether the $\mathrm{ABCD}$ method can predict the risk of gastric neoplasms, including gastric adenomas, as observed for gastric cancer. We aimed to devise a modified ABCD method for predicting gastric neoplasms.

Methods We reviewed 562 patients who had undergone upper gastrointestinal tract endoscopy and whose serum IgG anti-Helicobacter pylori antibody, gastrin, and pepsinogen (PG) I and PG II data were available. Patients were classified into the following four groups: H. pylori antibody negative and normal PG level (group A), H. pylori antibody positive and normal PG level (group B), H. pylori antibody positive and low PG level (group C), and $H$. pylori antibody negative and low PG level (group D).

Results The PG I/PG II ratio was lower in patients with gastric neoplasms than in patients without these lesions (gastric adenoma vs gastric cancer vs no neoplasm, $3.7 \pm 2.0$ vs $3.8 \pm 1.8$ vs $4.9 \pm 2.1, P<0.001$ ). The optimal cutoff values of the PG I/PG II ratio for predicting gastric neoplasms were 3.1 for $H$. pylori antibody negative patients and 4.1 for $H$. pylori antibody positive patients. A higher group grade was associated with a significantly
\end{abstract}

Electronic supplementary material The online version of this article (doi:10.1007/s10120-015-0473-4) contains supplementary material, which is available to authorized users.

C. H. Park - E. H. Kim - D. H. Jung $\cdot$ H. Chung

J. C. Park · S. K. Shin · S. K. Lee · Y. C. Lee ( $\square)$

Division of Gastroenterology, Department of Internal Medicine,

Severance Hospital, Institute of Gastroenterology, Yonsei

University College of Medicine, 50-1 Yonsei-ro, Seodaemun-gu,

Seoul 120-752, Republic of Korea

e-mail: leeyc@yuhs.ac higher proportion of gastric neoplasms [odds ratio (95\% confidence interval), group A, reference; group B, 1.783 (1.007-3.156); group C, 3.807 (2.382-6.085); and group D, $5.862(2.427-14.155)]$.

Conclusions The modified ABCD method using two different cutoff values according to the $H$. pylori antibody status was useful for predicting the presence of gastric neoplasms. This method might be a supplementary screening tool for both gastric adenoma and gastric cancer. However, further studies will be required to provide a definitive conclusion.

Keywords ABCD method - IgG anti-Helicobacter pylori antibody · Pepsinogen - Gastric cancer · Gastric adenoma

\section{Introduction}

Gastric cancer is one of the major causes of cancer-related death worldwide, and almost 990,000 cases of gastric cancer are detected annually [1]. The prognosis of patients with gastric cancer depends on the tumor stage [2-4]. In Korea and Japan, a mass screening program that uses upper gastrointestinal tract endoscopy and gastric fluoroscopy has been introduced for the early detection of gastric cancer [5, 6]. However, upper gastrointestinal tract endoscopy and gastric fluoroscopy are costly and inconvenient for people undergoing screening. In addition, gastric cancer is diagnosed in many patients without them undergoing periodic gastric cancer screening in Korea despite this nationwide cancer screening program [7].

In Japan, the ABCD screening method, which combines the detection of serum IgG anti-Helicobacter pylori antibody and the measurement of serum pepsinogen (PG) levels, has been developed for risk stratification of gastric 
cancer $[8,9]$. Because the ABCD screening method requires only blood sampling, it is much simpler and less invasive than upper gastrointestinal tract endoscopy or gastric fluoroscopy. This screening method is based on two well-known risk factors, namely, $H$. pylori infection and atrophic gastritis. The first risk factor, $H$. pylori infection, is evaluated on the basis of the IgG anti-H. pylori antibody status. The second risk factor, atrophic gastritis, is assessed by serum PG I and PG II levels. The serum PG status is usually defined as "atrophic" when both criteria of a serum PG I level of $70 \mathrm{ng} / \mathrm{mL}$ or lower and a PG I/PG II ratio of 3.0 or lower are simultaneously fulfilled, as proposed by Miki et al. [9, 10]. Then, individuals are classified into four groups as follows: (1) IgG anti-H. pylori antibody negative and normal PG level (group A), (2) IgG anti-H. pylori antibody positive and normal PG level (group B), (3) IgG anti- $H$. pylori antibody positive and atrophic PG status (group C), and (4) IgG anti-H. pylori antibody negative and atrophic PG status (group D). Previous studies demonstrated that the risk of gastric cancer was higher in group $\mathrm{C}$ or group D than in group A or group B $[8,11,12]$.

Although the $\mathrm{ABCD}$ screening method has been validated in some studies performed exclusively in Japan [8, 11-14], some questions remain unanswered. The first issue is the optimal PG cutoff value for determining atrophic gastritis. Several studies used the same criteria proposed by Miki et al. for defining atrophic PG status [8, 11-14]; however, Miki et al. [10] determined the PG cutoff value on the basis of endoscopically diagnosed atrophic gastritis rather than histologically confirmed atrophic gastritis. Therefore, the best PG cutoff value for optimal grouping in the $\mathrm{ABCD}$ method as well as for predicting histologic atrophic gastritis may differ somewhat from the value proposed by Miki et al. The second issue is whether the applicability of the ABCD method can be extended to predict the risk of gastric neoplasms including gastric adenomas as well as just gastric cancers. This may be an important issue at present because endoscopic resection has become increasingly used for the treatment of early gastric neoplasms including early gastric cancers and gastric adenomas [7].

To answer the aforementioned questions, we set two study aims. The first aim was to devise a modified ABCD method using the optimal PG cutoff value for gastric cancer risk stratification. The second aim was to ensure that the modified ABCD method could be applied for predicting gastric neoplasms rather than only gastric cancer.

\section{Methods}

\section{Patients}

We retrospectively reviewed consecutive patients who had undergone upper gastrointestinal tract endoscopy and whose serum IgG anti- $H$. pylori antibody, gastrin, and PG I and PG II data were available at Severance Hospital in Seoul, Korea, between May 2012 and August 2014. Patients with gastric neoplasms other than gastric adenoma and gastric cancer (i.e., gastric lymphoma, neuroendocrine tumor) were excluded from the study. Therefore, patients could be classified into three groups according to the disease status as follows: no neoplasm, gastric adenoma, and gastric cancer. We collected the following data from the patients' medical records: age, sex, presence of any gastric lesions and their location on upper gastrointestinal tract endoscopy, IgG anti-H. pylori antibody status, and the levels of serum gastrin, PG I, and PG II. This study was approved by the Institutional Review Board of Severance Hospital (IRB 4-2013-0722).

\section{Serological tests}

H. pylori infection was determined using enzyme-linked immunosorbent assay kits (Chorus Helicobacter pylori IgG; DIESSE Diagnostica Senese, Monteriggioni, Italy). Subjects with levels of $12.0 \mathrm{AU} / \mathrm{mL}$ or higher were classified as having $H$. pylori infection. Those with levels below $12.0 \mathrm{AU} / \mathrm{mL}$ were regarded as being $H$. pylori negative. The sensitivity and specificity of this assay for $H$. pylori infection were $89 \%$ and $100 \%$, respectively. The levels of PG I and PG II were measured using latex agglutination turbidimetric immunoassay kits (HiSens pepsinogen-I LTIA and HiSens pepsinogen-II LTIA; HBI, Anyang, Korea).

\section{Modification of the ABCD method}

Similarly to the original ABCD method, we also classified patients into four groups as follows: (1) IgG anti-H. pylori antibody negative and normal PG level (group A), (2) IgG anti- $H$. pylori antibody positive and normal PG level (group B), (3) IgG anti-H. pylori antibody positive and low PG level (group C), and (4) IgG anti-H. pylori antibody negative and low PG level (group D). Low PG level, however, was defined in a different manner. First, we determined the variables that significantly differed among patients according to the disease status. Among the variables of "both PG I and PG I/PG II ratio," "PG I," and "PG I/PG II ratio," only the significant PG variables were selected for determining low PG levels in the modified ABCD method. Second, the optimal PG cutoff value was identified using the receiver operating characteristic (ROC) curve and the Youden index for predicting the presence of gastric neoplasms. Third, we determined the PG cutoff values in the $\operatorname{IgG}$ anti- $H$. pylori antibody positive and $\operatorname{IgG}$ anti-H. pylori antibody negative subgroups because $H$. pylori infection affects gastric atrophy and PG level, and 
reliable PG cutoff values may differ according to the $H$. pylori infection status [15-17].

\section{Statistical analysis}

Continuous variables are presented as the mean with the standard deviation and were compared by using the $t$ test or analysis of variance. When the significance was identified via analysis of variance, the Bonferroni correction was used for post hoc analysis. Categorical variables are presented as the sample number with the proportion and were compared by using the $\chi^{2}$ test or Fisher's exact test. To determine the PG cutoff values, the ROC curve and the Youden index were used. In addition, the Cochran-Armitage trend test was performed for trend analysis of the proportion of gastric neoplasms according to the $\mathrm{ABCD}$ grouping. Finally, logistic regression analysis was performed to evaluate whether the modified ABCD method could predict the presence of gastric neoplasms under adjustment for possible confounding variables, including age and sex. A $P$ value below 0.05 was considered significant for group comparisons. All statistical analyses were performed using the statistical software program SPSS for Windows (version 18.0; SPSS, Chicago, IL, USA), except for the Cochran-Armitage trend test, for which the analysis was performed using R (version 2.15.3; R Foundation for Statistical Computing, Vienna, Austria).

\section{Results}

Baseline characteristics

A total of 584 patients who had undergone upper gastrointestinal tract endoscopy and whose serum IgG anti- $H$. pylori antibody, gastrin, and PG I and PG II data were available were identified. Of these, 15 patients with extranodal marginal zone B-cell lymphoma and seven patients with gastric neuroendocrine tumors were excluded. The remaining 562 patients were retrospectively analyzed in the study. The mean age of the patients was 59 years, and $48.6 \%$ of the patients were men (Table 1). Gastric adenomas and cancers were detected in 51 patients $(9.1 \%)$ and 131 patients $(23.3 \%)$, respectively. The remaining 380 patients $(67.6 \%)$ did not exhibit gastric adenoma or gastric cancer on endoscopy, and these patients formed the no neoplasm (control) group.

Laboratory tests according to the disease status

The patient demographics and the results of laboratory tests according to disease status are presented in Table 2. Patients in the gastric adenoma and gastric cancer groups
Table 1 Baseline patient and lesion characteristics

\begin{tabular}{|c|c|}
\hline Variable & Value \\
\hline No. of patients & 562 \\
\hline Mean age \pm SD (years) & $58.5 \pm 12.5$ \\
\hline \multicolumn{2}{|l|}{ Sex } \\
\hline Male & $273(48.6 \%)$ \\
\hline Female & $289(51.4 \%)$ \\
\hline \multicolumn{2}{|l|}{ Disease } \\
\hline No neoplasm ${ }^{\mathrm{a}}$ & $380(67.6 \%)$ \\
\hline Gastric adenoma & $51(9.1 \%)$ \\
\hline Gastric cancer & $131(23.3 \%)$ \\
\hline \multicolumn{2}{|l|}{ Depth of invasion ${ }^{\mathrm{b}}$} \\
\hline Mucosa & $79(60.3 \%)$ \\
\hline Submucosa & $21(16.0 \%)$ \\
\hline Proper muscle & $11(8.4 \%)$ \\
\hline Subserosa & $6(4.6 \%)$ \\
\hline Serosa & $14(10.7 \%)$ \\
\hline \multicolumn{2}{|l|}{ Histology ${ }^{\mathrm{b}}$} \\
\hline Differentiated & $80(61.1 \%)$ \\
\hline Undifferentiated & $51(38.9 \%)$ \\
\hline \multicolumn{2}{|l|}{ Tumor location $^{\mathrm{c}}$} \\
\hline Upper third & $17(9.3 \%)$ \\
\hline Middle third & $53(29.1 \%)$ \\
\hline Lower third & $112(61.5 \%)$ \\
\hline \multicolumn{2}{|l|}{$S D$ standard deviation } \\
\hline \multicolumn{2}{|c|}{${ }^{a}$ This category included patients with dyspepsia } \\
\hline \multicolumn{2}{|c|}{$\begin{array}{l}\text { b This variable was calculated on the basis of the number of patients } \\
\text { with gastric cancer }\end{array}$} \\
\hline
\end{tabular}

were older than those in the no neoplasm group (gastric adenoma vs gastric cancer vs no neoplasm, $64.5 \pm$ 8.8 years vs $60.4 \pm 11.0$ years vs $57.0 \pm 13.0$ years, $P<0.001)$. In addition, the proportion of men was higher in the gastric adenoma and gastric cancer groups than in the no neoplasm group (gastric adenoma vs gastric cancer vs no neoplasm, $64.74 \%$ vs $71.84 \%$ vs $38.4 \%, P<0.001$ ).

Serum gastrin levels did not differ among the three groups $(P=0.312)$. PG I levels also did not differ among the three groups $(P=0.102)$. Although the PG II level was higher in the gastric cancer group than in the no neoplasm group $(P<0.001)$, it did not differ between the gastric adenoma and no neoplasm groups $(P>0.999)$. Both the gastric adenoma group and the gastric cancer group displayed significantly lower values of the PG I/PG II ratio than did the no neoplasm group (gastric adenoma vs gastric cancer vs no neoplasm, $3.7 \pm 2.0$ vs $3.8 \pm 1.8$ vs $4.9 \pm 2.1, P<0.001)$. In addition, the prevalence of $\operatorname{IgG}$ anti- $H$. pylori antibody positive status was higher in the gastric adenoma and gastric cancer groups than into the no 
Table 2 Demographics and results of laboratory tests according to the disease status

\begin{tabular}{|c|c|c|c|c|c|c|c|}
\hline \multirow[t]{2}{*}{ Variable } & \multirow{2}{*}{$\begin{array}{l}\text { No neoplasm } \\
(n=380)\end{array}$} & \multirow{2}{*}{$\begin{array}{l}\text { Gastric } \\
\text { adenoma } \\
(n=51)\end{array}$} & \multirow{2}{*}{$\begin{array}{l}\text { Gastric } \\
\text { cancer } \\
(n=131)\end{array}$} & \multirow[t]{2}{*}{$P$} & \multicolumn{3}{|l|}{$P^{\mathrm{a}}$} \\
\hline & & & & & $\begin{array}{l}\text { No neoplasm vs } \\
\text { gastric adenoma }\end{array}$ & $\begin{array}{l}\text { No neoplasm vs } \\
\text { gastric cancer }\end{array}$ & $\begin{array}{l}\text { Gastric adenoma } \\
\text { vs gastric cancer }\end{array}$ \\
\hline Age (years) & $57.0 \pm 13.0$ & $64.5 \pm 8.8$ & $60.4 \pm 11.0$ & $<0.001$ & $<0.001$ & 0.019 & 0.131 \\
\hline Sex & & & & $<0.001$ & & & \\
\hline Male & $146(38.4 \%)$ & $33(64.7 \%)$ & $94(71.8 \%)$ & & & & \\
\hline Female & $234(61.6 \%)$ & $18(35.3 \%)$ & $37(28.2 \%)$ & & & & \\
\hline Gastrin $(\mathrm{pg} / \mathrm{mL})$ & $82.3 \pm 153.8$ & $115.1 \pm 132.5$ & $97.9 \pm 196.3$ & 0.312 & & & \\
\hline PG I (ng/mL) & $64.9 \pm 40.3$ & $58.6 \pm 52.8$ & $72.6 \pm 51.1$ & 0.102 & & & \\
\hline PG II (ng/mL) & $15.5 \pm 11.7$ & $16.4 \pm 11.4$ & $20.2 \pm 12.7$ & $<0.001$ & $>0.999$ & $<0.001$ & 0.149 \\
\hline PG I/PG II ratio & $4.9 \pm 2.1$ & $3.7 \pm 2.0$ & $3.8 \pm 1.8$ & $<0.001$ & $<0.001$ & $<0.001$ & $>0.999$ \\
\hline $\begin{array}{l}\text { IgG anti- } \\
\text { Helicobacter pylori } \\
\text { antibody }\end{array}$ & & & & $<0.001$ & & & \\
\hline Negative & $192(50.5 \%)$ & $17(33.3 \%)$ & $35(26.7 \%)$ & & & & \\
\hline Positive & $188(49.5 \%)$ & $34(66.7 \%)$ & $96(73.3 \%)$ & & & & \\
\hline
\end{tabular}

Values are presented as the mean with the standard deviation or as the number with the proportion.

$P G$ pepsinogen

a Results of post hoc analysis using the Bonferroni correction

neoplasm group (gastric adenoma vs gastric cancer vs no neoplasm, $66.7 \%$ vs $73.3 \%$ vs $49.5 \%, P<0.001)$.

Comparison of laboratory tests according to the IgG anti-H. pylori antibody status

Figure 1 shows the results of laboratory tests for the IgG antiH. pylori antibody negative and the IgG anti-H. pylori antibody positive subgroups in the no neoplasm, gastric adenoma, and gastric cancer groups. Serum gastrin levels did not differ between the IgG anti-H. pylori antibody negative and IgG anti-H. pylori antibody positive subgroups (Fig. 1a). Although serum PG I levels were higher in the IgG anti- $H$. pylori antibody positive subgroup than in the IgG anti- $H$. pylori antibody negative subgroup in patients who had neither gastric adenoma nor gastric cancer, it did not differ according to the IgG anti-H. pylori antibody status in patients with either the gastric adenoma group or the gastric cancer group (Fig. 1b). Serum PG II levels, however, were higher in the IgG anti-H. pylori antibody positive subgroup than in the IgG anti- $H$. pylori antibody negative subgroup in all three groups (Fig. 1c). In addition, the PG I/PG II ratio was lower in the IgG anti-H. pylori antibody positive subgroup than in the IgG anti-H. pylori antibody negative subgroup in all three groups (Fig. 1d).

Determination of cutoff values for the PG I/PG II ratio

Because PG I levels did not differ among the no neoplasm, gastric adenoma, and gastric cancer groups, as shown in Table 2, we chose only the PG I/PG II ratio rather than
PG I level as the variable for determining low PG levels in the modified ABCD method. In patients with IgG anti- $H$. pylori antibody negative status, the optimal cutoff value for the PG I/PG II ratio was 3.1 (Fig. 2a). The optimal cutoff values for the PG I/PG II ratio for predicting gastric neoplasms were 3.1 for patients with IgG anti-H. pylori antibody negative status and 4.1 for patients with IgG anti- $H$. pylori antibody positive status (Fig. 2b).

Relationship between gastric neoplasms and the modified ABCD grouping

Using the cutoff values for the PG I/PG II ratio derived from the ROC curves and the Youden index, we classified the patients into four groups as shown in Table 3. In patients without gastric neoplasms, the numbers of patients in groups A, B, C, and D were 179, 76, 112, and 13, respectively. In patients with gastric neoplasms, the numbers of patients in groups A, B, C, and D were 37, 32, 98, and 15 , respectively. Of the 182 patients with gastric neoplasms, $113(62.1 \%)$ were in group C or group D. The Cochran-Armitage trend test confirmed that a higher group grade was associated with a significantly higher proportion of gastric neoplasms $\left(P_{\text {trend }}<0.001\right)$.

To compare the screening capacity between the original ABCD method and the modified ABCD method, numbers of patients who were classified by the original ABCD grouping are shown in Table S1. In patients with gastric neoplasms, the numbers of patients in groups $\mathrm{A}, \mathrm{B}, \mathrm{C}$, and D were 40, 86, 44, and 12, respectively. Compared with the classification by the modified ABCD method, fewer 


\section{a}

$\mathrm{pg} / \mathrm{mL}$

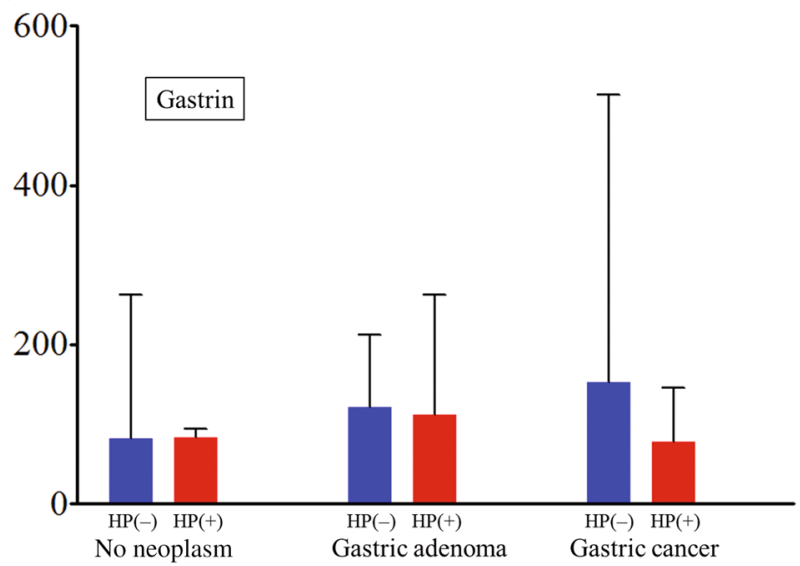

C

$\mathrm{ng} / \mathrm{mL}$

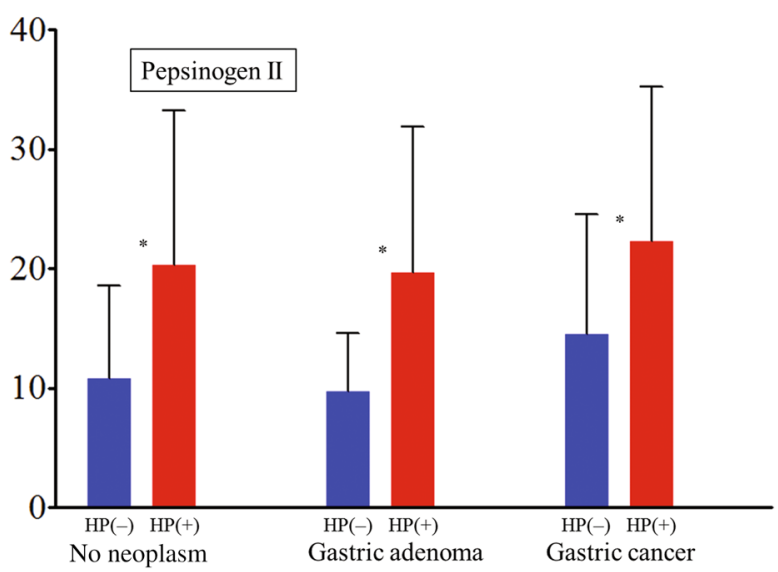

b

$\mathrm{ng} / \mathrm{mL}$

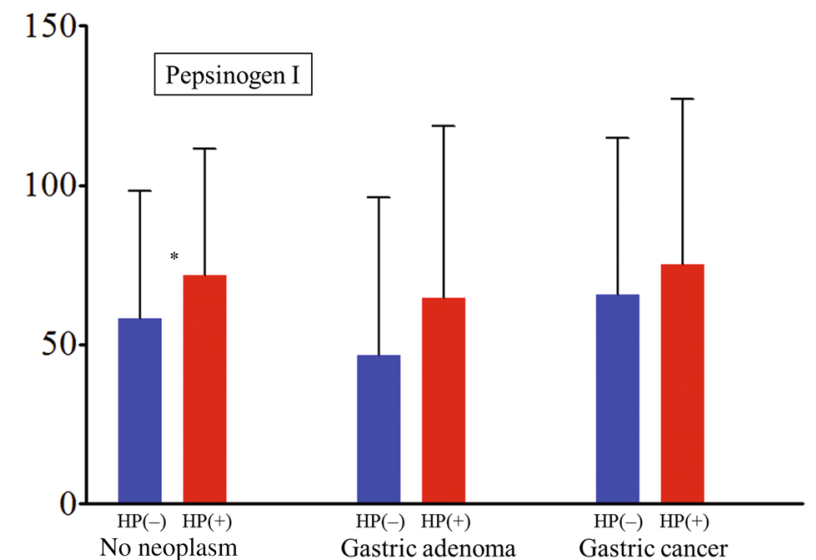

d

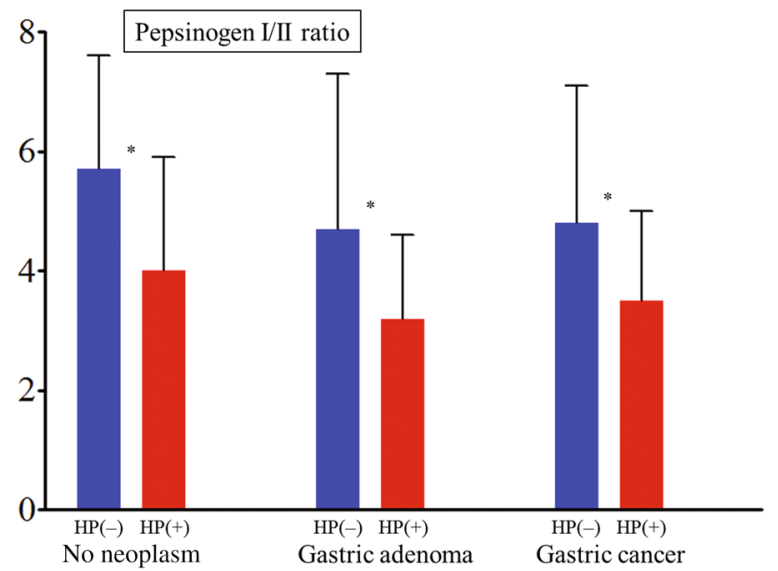

Fig. 1 Laboratory tests for gastrin (a), pepsinogen (PG) I (b), PG II (c), and the PG I/PG II ratio (d) according to the IgG anti-Helicobacter pylori antibody status. $H P(+)$ and $H P(-)$ denote IgG anti- $H$. pylori antibody positive and IgG anti- $H$. pylori antibody negative, respectively

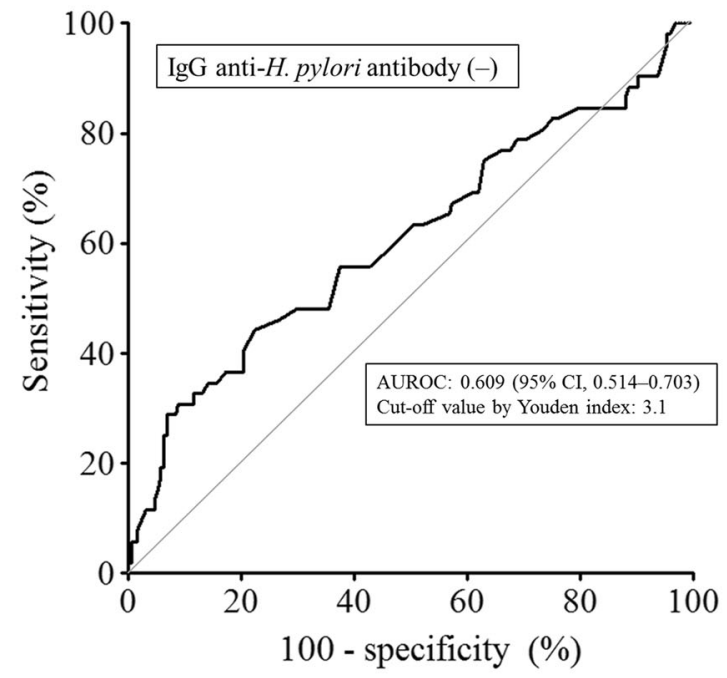

Fig. 2 Receiver operating characteristic curves for predicting gastric neoplasms based on the pepsinogen (PG) I/PG II ratio in patients with $\mathrm{IgG}$ anti-Helicobacter pylori antibody negative status (a) and IgG

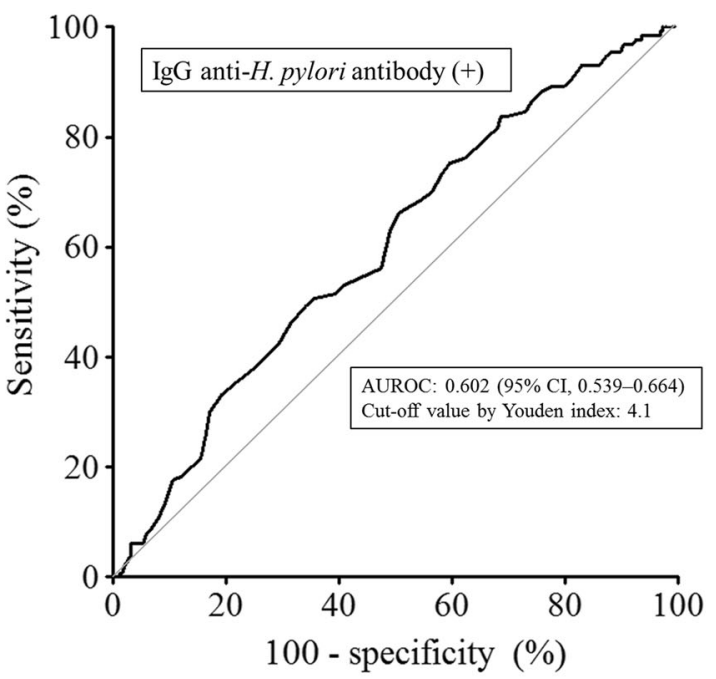

anti-H. pylori antibody positive status (b). Gastric neoplasms included gastric adenoma and gastric cancer. AUROC area under the receiver operating characteristic curve, $C I$ confidence interval 
Table 3 The relationship between gastric neoplasms and the modified ABCD grouping

\begin{tabular}{lllll}
\hline Variable & $\begin{array}{l}\text { Group A: IgG anti- } \\
\text { Helicobacter pylori } \\
\text { antibody negative and PG I/ } \\
\text { PG II ratio }>3.1\end{array}$ & $\begin{array}{l}\text { Group B: IgG anti-H. } \\
\text { pylori antibody positive } \\
\text { and PG I/PG II ratio } \\
>4.1\end{array}$ & $\begin{array}{l}\text { Group C: IgG anti- } H . \\
\text { pylori antibody positive } \\
\text { and PG I/PG II ratio } \\
\leq 4.1\end{array}$ & $\begin{array}{l}\text { Group D: IgG anti- } H . \\
\text { pylori antibody negative } \\
\text { and PG I/PG II ratio } \\
\leq 3.1\end{array}$ \\
\hline $\begin{array}{l}\text { No } \\
\text { neoplasms } \\
(n=380)\end{array}$ & $179(47.1 \%)$ & $76(20.0 \%)$ & $112(29.5 \%)$ & $13(3.4 \%)$ \\
$\begin{array}{c}\text { Gastric } \\
\text { neoplasms } \\
(n=182)\end{array}$ & $37(20.3 \%)$ & $32(17.6 \%)$ & $98(53.8 \%)$ & $15(8.2 \%)$ \\
\hline
\end{tabular}

Gastric neoplasms included gastric adenoma and gastric cancer

$P G$ pepsinogen

Table 4 Multiple logistic regression model for predicting the presence of gastric neoplasms

\begin{tabular}{|c|c|c|c|c|}
\hline Variable & Number & Gastric neoplasms & $\mathrm{OR}^{\mathrm{a}}$ & $P$ \\
\hline Age (years) & & & $1.030(1.013-1.048)$ & 0.001 \\
\hline \multicolumn{5}{|l|}{ Sex } \\
\hline Male & 273 & $127(46.5 \%)$ & $3.550(2.370-5.319)$ & $<0.001$ \\
\hline Female & 289 & $55(19.0 \%)$ & 1.000 & \\
\hline \multicolumn{5}{|l|}{ IgG anti-Helicobacter pylori antibody status and PG I/PG II ratio } \\
\hline Group A: IgG anti-H. pylori antibody negative and PG I/PG II ratio $>3.1$ & 216 & $29(13.4 \%)$ & 1.000 & \\
\hline Group B: IgG anti-H. pylori antibody positive and PG I/PG II ratio $>4.1$ & 108 & $21(19.4 \%)$ & $1.783(1.007-3.156)$ & 0.047 \\
\hline Group C: IgG anti-H. pylori antibody positive and PG I/PG II ratio $\leq 4.1$ & 210 & $109(51.9)$ & $3.807(2.382-6.085)$ & $<0.001$ \\
\hline Group D: IgG anti-H. pylori antibody negative and PG I/PG II ratio $\leq 3.1$ & 28 & $23(82.1 \%)$ & $5.862(2.427-14.155)$ & $<0.001$ \\
\hline
\end{tabular}

Gastric neoplasms included gastric adenoma and gastric cancer

$O R$ odds ratio, $P G$ pepsinogen

a The $95 \%$ confidence interval is given in parentheses

patients with gastric neoplasms were in group $\mathrm{C}$ or group $\mathrm{D}$ [original ABCD method vs modified ABCD method, 56 $(30.8 \%)$ vs $113(62.1 \%), P<0.001]$.

Finally, we created a multiple logistic regression model to determine the relationship between the ABCD grouping and the proportion of gastric neoplasms after adjustment for confounding variables, including age and sex (Table 4). The prevalence of gastric neoplasms in groups B, C, and D was higher than that in group A [odds ratio (95\% confidence interval), group $\mathrm{B}, 1.783$ (1.007-3.156); group $\mathrm{C}$, 3.807 (2.382-6.085); and group D, 5.862 (2.427-14.155)].

\section{Discussion}

The ABCD screening method, which was developed in Japan, can easily be used for risk stratification of gastric cancer because it is simple and less invasive compared with upper gastrointestinal tract endoscopy or gastric fluoroscopy [9]. Considering that gastric cancer is diagnosed in many patients without them undergoing periodic gastric cancer screening in Korea despite there being a national gastric cancer screening program [7], the ABCD method may have a role in gastric cancer screening in regions in which the incidence of gastric cancer is high. Although some studies demonstrated that a higher group grade in the ABCD method was associated with a higher risk of gastric cancer [8, 11, 12], all of those studies focused on only gastric cancer rather than gastric adenoma. Although screening for gastric cancer is important, screening for gastric adenoma is also important. Correa [18] postulated that gastric cancer may be induced from chronic gastritis via intestinal metaplasia and dysplasia. Recently, a dysplasia-carcinoma sequence has been reported to be associated with the development of intestinal-type gastric cancer [8, 19, 20]. When gastric dysplasia is detected by screening, curative removal via endoscopic resection is possible.

Our study has merits for being the first study to demonstrate that the presence of gastric neoplasms, including gastric adenoma, could be predicted by the IgG anti-H. pylori antibody status and PG I/PG II ratio. In patients with a higher group grade in the modified ABCD method, more careful and strict screening may be required 
for the early diagnosis of both gastric adenoma and gastric cancer.

Another advantage of the modified ABCD method is the use of two different cutoff values for the PG I/PG II ratio according to the IgG anti-H. pylori antibody status. Among the four groups in the ABCD method, the risk of group A is distinct from that of group D despite the identical IgG antiH. pylori antibody status between the two groups. IgG anti$H$. pylori antibody negative status in group A means that patients have never been infected with $H$. pylori. In contrast, IgG anti-H. pylori antibody negative status in group D implies prior $H$. pylori infection because $H$. pylori does not survive well in the atrophic or intestinal metaplasia mucosa [12]. Compared with groups A and D, a relatively similar severity of the gastric mucosa was expected in terms of gastric atrophy between groups $\mathrm{B}$ and $\mathrm{C}$ because significant proportions of patients in both groups may be currently infected with $H$. pylori. In other words, the distribution of the PG I/PG II ratio may differ between patients with IgG anti- $H$. pylori antibody negative status (groups A and D) and those with IgG anti-H. pylori antibody positive status (groups B and C). In our study, as expected, the optimal cutoff value for the PG I/PG II ratio did differ between the patients with IgG anti- $H$. pylori antibody negative status and those with IgG anti-H. pylori antibody positive status. This new approach may help to increase the screening capacity of the ABCD method for gastric neoplasms.

Although this study was the first to evaluate whether gastric neoplasms could be predicted by the IgG anti- $H$. pylori antibody status and serum PG levels, there are several limitations. First, a few patients who had undergone upper gastrointestinal tract endoscopy were not included because their serum IgG anti-H. pylori antibody, gastrin, or PG I and PG II data were unavailable, and a possible selection bias could be a concern. However, we think that this selection bias may not be serious, because tests for serum IgG anti- $H$. pylori antibody, gastrin, and PG I and PG II had been performed only for individuals who had undergone gastrointestinal tract endoscopy for screening purposes or patients in whom gastric neoplasms had been diagnosed. Second, this study was based on nonrepresentative groups of patients. Our results identified 380 patients $(67.6 \%), 51$ patients $(9.1 \%)$, and 131 patients $(23.3 \%)$ with no neoplasm, gastric adenoma, and gastric cancer, respectively; however, this distribution did not represent the prevalence of gastric adenoma and gastric cancer in the screening population. A populationbased cohort study is required to prove the usefulness of the modified ABCD method for gastric neoplasm screening. The third limitation is the cross-sectional design of the study. The study did not include follow-up data; therefore, we could evaluate only the predictive accuracy concerning the presence of gastric neoplasms rather than the risk of gastric cancer development in the future. Our study design, however, helped to include a larger number of gastric cancers compared with previous studies [8, 11-14]. Fourth, data on histologic atrophic gastritis were unavailable in the study. Although we determined the cutoff values for the PG I/PG II ratio using ROC curves for predicting the presence of gastric neoplasms, they should be determined ideally using ROC curves to predict the presence of histologically confirmed atrophic gastritis. To remedy this limitation, we plan to perform a prospective study on the optimal PG cutoff values for histologic atrophic gastritis. Finally, although we demonstrated the usefulness of the modified ABCD method using multiple logistic regression models, external validation is mandatory to provide a definitive conclusion. Further studies on the modified ABCD method are expected.

Despite these limitations, our data may form the basis of a system to suggest a new screening strategy for gastric cancer. The modified ABCD method using two different cutoff values according to the IgG anti- $H$. pylori antibody status was useful for predicting the presence of gastric neoplasms. We believe that the modified ABCD method can be a supplementary screening tool for both gastric adenoma and gastric cancer. However, further studies will be required to provide a definitive conclusion.

\section{References}

1. Jemal A, Bray F, Center MM, Ferlay J, Ward E, Forman D. Global cancer statistics. CA Cancer J Clin. 2011;61:69-90.

2. Yokota T, Kunii Y, Teshima S, Yamada Y, Saito T, Takahashi M, et al. Significant prognostic factors in patients with early gastric cancer. Int Surg. 2000;85:286-90.

3. Siewert JR, Böttcher K, Stein HJ, Roder JD. Relevant prognostic factors in gastric cancer: ten-year results of the German Gastric Cancer Study. Ann Surg. 1998;228:449-61.

4. Lee HJ, Yang HK, Ahn YO. Gastric cancer in Korea. Gastric Cancer. 2002;5:177-82.

5. Nishi M, Ishihara S, Nakajima T, Ohta K, Ohyama S, Ohta H. Chronological changes of characteristics of early gastric cancer and therapy: experience in the Cancer Institute Hospital of Tokyo, 1950-1994. J Cancer Res Clin Oncol. 1995;121:535-41.

6. National cancer control programs in Korea. J Korean Med Sci. 2007;22(Suppl):S3-4.

7. Park CH, Kim EH, Chung H, Lee H, Park JC, Shin SK, et al. The optimal endoscopic screening interval for detecting early gastric neoplasms. Gastrointest Endosc. 2014;80:253-9.

8. Ohata H, Kitauchi S, Yoshimura N, Mugitani K, Iwane M, Nakamura $\mathrm{H}$, et al. Progression of chronic atrophic gastritis associated with Helicobacter pylori infection increases risk of gastric cancer. Int J Cancer. 2004;109:138-43.

9. Miki K. Gastric cancer screening by combined assay for serum anti-Helicobacter pylori IgG antibody and serum pepsinogen levels_-"ABC method". Proc Jpn Acad Ser B Phys Biol Sci. 2011;87:405-14.

10. Miki K, Ichinose M, Shimizu A, Huang SC, Oka H, Furihata C, et al. Serum pepsinogens as a screening test of extensive chronic gastritis. Gastroenterol Jpn. 1987;22:133-41. 
11. Watabe H, Mitsushima T, Yamaji Y, Okamoto M, Wada R, Kokubo T, et al. Predicting the development of gastric cancer from combining Helicobacter pylori antibodies and serum pepsinogen status: a prospective endoscopic cohort study. Gut. 2005;54:764-8.

12. Mizuno S, Miki I, Ishida T, Yoshida M, Onoyama M, Azuma T, et al. Prescreening of a high-risk group for gastric cancer by serologically determined Helicobacter pylori infection and atrophic gastritis. Dig Dis Sci. 2010;55:3132-7.

13. Yamaji Y, Mitsushima T, Ikuma H, Okamoto M, Yoshida H, Kawabe T, et al. Inverse background of Helicobacter pylori antibody and pepsinogen in reflux oesophagitis compared with gastric cancer: analysis of 5732 Japanese subjects. Gut. 2001;49:335-40.

14. Kudo T, Kakizaki S, Sohara N, Onozato Y, Okamura S, Inui Y, et al. Analysis of ABC (D) stratification for screening patients with gastric cancer. World J Gastroenterol. 2011;17:4793-8.

15. Lorente S, Doiz O, Trinidad Serrano M, Castillo J, Lanas A. Helicobacter pylori stimulates pepsinogen secretion from isolated human peptic cells. Gut. 2002;50:13-8.
16. Kim HY, Kim N, Kang JM, Park YS, Lee DH, Kim YR, et al. Clinical meaning of pepsinogen test and Helicobacter pylori serology in the health check-up population in Korea. Eur $\mathbf{J}$ Gastroenterol Hepatol. 2009;21:606-12.

17. Bölükbaş C, Bölükbaş F, Ovünç O, Kiliç G, Dalay R, Güven H, et al. Relationship between Helicobacter pylori status and serum pepsinogens as serologic markers in atrophic gastritis. Turk $\mathbf{J}$ Gastroenterol. 2006;17:172-6.

18. Correa P. A human model of gastric carcinogenesis. Cancer Res. 1988;48:3554-60.

19. Vieth M, Stolte M. Elevated risk for gastric adenocarcinoma can be predicted from histomorphology. World J Gastroenterol. 2006;12:6109-14.

20. Adamu MA, Weck MN, Gao L, Brenner H. Incidence of chronic atrophic gastritis: systematic review and meta-analysis of followup studies. Eur J Epidemiol. 2010;25:439-48. 